\title{
Development of internal stress measurement technique for Ni electroforming using Shack-Hartmann sensor
}

\author{
Takehiro Kume*, Satoru Egawa*, Hidekazu Mimura* \\ (Received Jan. 15, 2016) \\ * Department of Precision Engineering, Graduate School of Engineering, The University of Tokyo, 7-3-1 \\ Hongo, Bunkyo-ku, Tokyo 113-8656, Japan
}

\begin{abstract}
The performance of an in situ internal stress measurement technique using a Shack-Hartmann sensor (SHS) is examined during nickel electrodeposition in concentrated nickel sulfamate solution. The stress is calculated from the change in the curvature of a test plate bent by stress. The curvature is measured by monitoring the wavefront of an expanded laser beam reflected at the substrate using an SHS. Applying the proposed method, curvature measurements were conducted and the repeatability and accuracy were estimated. In situ stress measurement with a current density of $2.1 \mathrm{~mA} / \mathrm{cm}^{2}$ revealed a repeatability of approximately $1 \mathrm{MPa}$ when the thickness of the nickel layer was $19 \mu \mathrm{m}$.
\end{abstract}

Key words: electrodeposition, nickel sulfamate, internal stress, in situ observation

\section{INTRODUCTION}

Electroforming is a replication technique involving the electrodeposition of a metal on a master surface and the removal of the master as shown in Fig. 1. Electroforming can replicate the shape and microstructure of a master surface with high accuracy. Thus, this method has played an important role in industry in the fabrication of microelectromechanical systems (MEMS), precision molding and precision optics ${ }^{1)}$, 2 . Because of its shape replication accuracy, it is applied to the fabrication of high-precision mirrors used for x-ray microscopy that require shape accuracy of $1 \mathrm{~nm}$ order $^{3}$. However, further improvement of the replication accuracy is still needed.

The replication accuracy is limited by internal stress resulting from the electrodeposition of the metal because it causes unwanted deformation after the removal of the master. The internal stress depends on the electrolyte composition, temperature and current density. In general, to fabricate a precision product by electroforming, the internal stress is measured under several current densities beforehand and the appropriate current

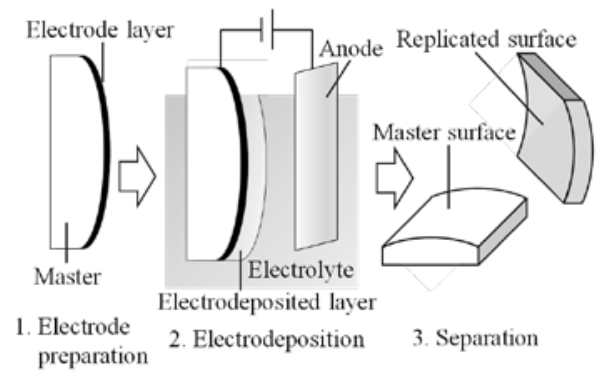

Fig. 1. Electroforming. density is adopted to minimize the stress in the product. Therefore, a reliable method for internal stress measurement with high accuracy is needed to realize replication accuracy of $1 \mathrm{~nm}$ order.

Several stress measurement techniques have been developed since early in the 20 th century ${ }^{4), 5)}$. In particular, in situ stress measurement methods in which the stress is calculated from the change in the curvature of a test plate are commonly used in electroforming. The use of a spiral contractometer is one of the most common methods in industry because of its simplicity ${ }^{6}$. However, it is not suitable for precise measurements because of its low accuracy. Laser deflection methods are more accurate and frequently used when high accuracy is required. During measurement, the position of a laser beam reflected at a test plate is recorded by a detector and the curvature is estimated by the change in the position caused by the deformation of the test plate. Another accurate measurement method is the use of a multibeam optical sensor (MOS). Since a multibeam and CCD camera are used instead of a single laser beam and a position-sensitive detector, MOS measurements are less sensitive to vibrations and are applied for observation in both vacuum and liquid environments $^{\text {7) }}$-9)

Recently, we have developed an advanced method for in situ stress measurement during electrodeposition that uses a single expanded laser beam and a Shack-Hartmann sensor (SHS, SHSLab, Optocraft ${ }^{10)}$. An SHS is a wavefront sensor that consists of a microlens array and a CCD camera. An incident wavefront forms an array of spots at the CCD camera after refraction at the microlens array, and the shape of the wavefront is estimated from the positions of the spots. The measured wavefront contains information on the shape of the 
entire area where the beam is reflected and stress measurement with high accuracy, high stability and repeatability is expected.

In this study, using a stress measurement apparatus including an SHS, the accuracy and repeatability of measurements are examined under practical conditions for highly accurate electroforming. Since shape replication with accuracy of sub-100 nm order has been reported at room temperature, every experiment was conducted at room temperature ${ }^{3)}$.

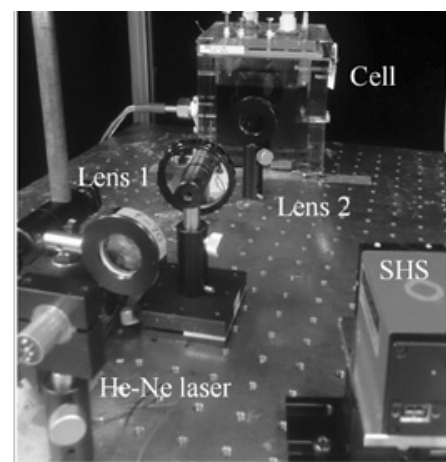

(a) Photograph of apparatus.

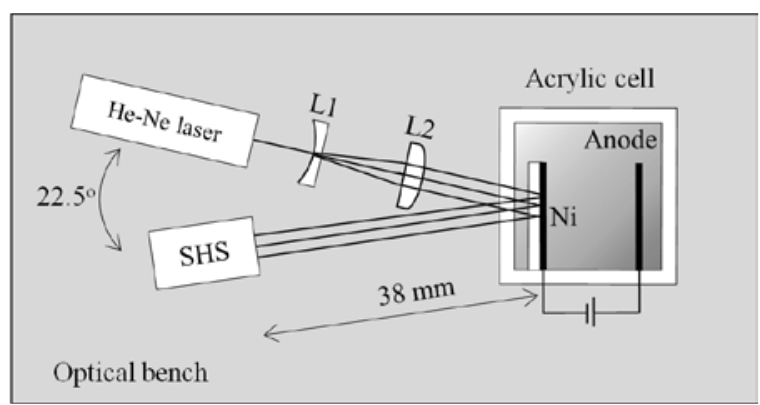

(b) Top view of optical system.

Fig. 2. Stress measurement apparatus.

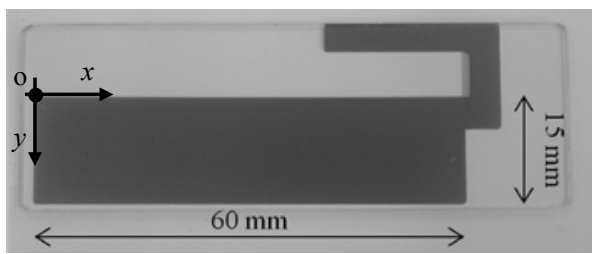

(a) Photograph of substrate.
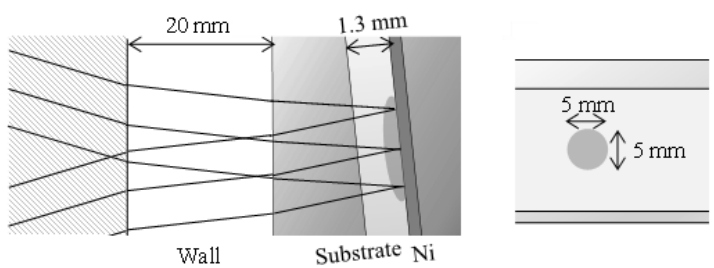

(b) Reflected area

Fig. 3. Substrate and evaporated Ni layer.

\section{EXPERIMENTAL SETUP}

\subsection{Construction}

Fig. 2 shows the optical components of the stress measurement setup. An evaporated nickel layer on a glass substrate, as shown in Fig. 3, is used as a cathode. The internal stress in an electrodeposited layer is measured by monitoring the curvature of the glass substrate. First, the substrate is illuminated by a He-Ne laser expanded by two lenses through a transparent electrolytic cell The wavefront of the light reflected at the substrate surface is monitored by an SHS. The wavefront before electrodeposition is obtained as a reference wavefront and it is subtracted from the measured wavefront during electrodeposition. Fig. 4 shows a wavefront measured by the SHS during electrodeposition using the setup. This wavefront suggests that the substrate is distorted into a spherical shape by internal stress. Thus, the curvature of the substrate is calculated from that of the least-squares sphere of the measured wavefront by ray tracing, assuming that the stress is evenly distributed. Finally, the internal stress is calculated from the strength characteristics of the substrate and nickel.

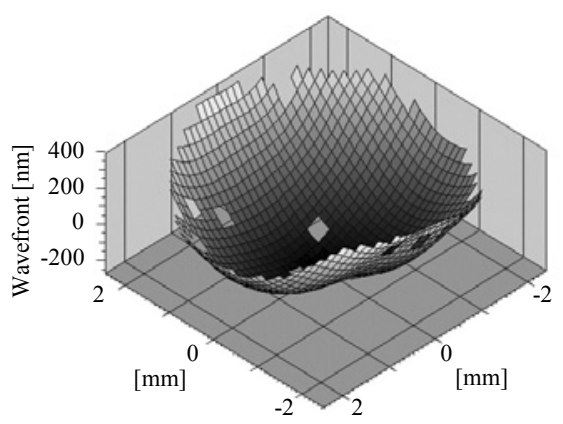

Fig. 4. Measured wavefront reflected at substrate deformed by internal stress.

\subsection{Calculation}

In general, electroformed products have a thickness of $100 \mu \mathrm{m}$ to $1 \mathrm{~mm}$ order to prevent plastic deformation resulting from the separation force. From a practical viewpoint, the thickness of the deposited layer in stress measurement should be at least $10 \mu \mathrm{m}$ order. Compared with the glass substrate, the thickness of the deposited layer cannot be disregarded and the thin-film approximation is not satisfied.

In this study, stress is calculated by assuming that the total of the strain energy and bending energy $U$ is minimal. The strain energy of nickel $U_{l}$, the bending energy of nickel $U_{2}$, the strain energy of the substrate $U_{3}$ and the bending energy of the substrate $U_{4}$ are expressed as follows: 


$$
\begin{aligned}
& U_{1}=\frac{A_{N} E_{N}(x-X)^{2}}{2 X}, \\
& U_{2}=\frac{E_{N} I_{N} L}{2 \kappa_{N}^{2}}, \\
& U_{3}=\frac{A_{S} E_{S}(l-L)^{2}}{2 L}, \\
& U_{4}=\frac{E_{S} I_{S} L}{2 \kappa_{S}^{2}},
\end{aligned}
$$

where $A_{N}\left(A_{S}\right)$ is the cross-sectional area of the nickel layer (substrate), $E_{N}\left(E_{S}\right)$ is the Young's modulus of nickel (soda-lime glass), $x(l)$ is the length of the electrodeposited layer (substrate) along the neutral axis during electrodeposition, $L$ is the length of the electrode layer and the substrate along the neutral axis before electrodeposition, $X$ is the length of the electrodeposited layer along the neutral axis when the stress is released, $I_{N}\left(I_{S}\right)$ is the second moment of the area of the electrodeposited layer (substrate) and $\kappa_{N}\left(\kappa_{S}\right)$ is the curvature of the electrodeposited nickel layer (glass substrate) as illustrated in Fig. 5. The thickness of the electrodeposited layer is calculated from the current density and deposition time using Faraday's law of electrolysis. The strain distribution in the substrate along the $z$-axis $\varepsilon(z)$ is assumed to be linear and it is expressed as

$$
\varepsilon(z)=k z+a,
$$

where $k$ and $a$ are constants.

Assuming $d U / d x=0$, the geometrical relations and boundary conditions, the stress in the nickel layer during electrodeposition $\sigma$ is obtained as

$$
\begin{aligned}
\sigma= & E_{N} \frac{x-X}{X} \\
= & \frac{E_{N}}{A_{N}}\left\{I_{N}\left(\frac{1}{1-\left(\frac{d+t}{2}\right) \kappa_{S}}\right)^{3}+\frac{4}{3} \frac{E_{S} I_{S}}{E_{N}}\right\} \\
& \cdot \frac{6 \kappa_{S}}{4 d+3 t-d(d+t) \kappa_{S}},
\end{aligned}
$$

where the thickness of the electrodeposited layer (substrate) is $t(d)$.

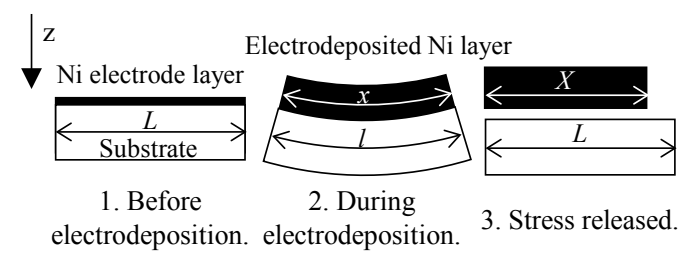

Fig. 5. Deformation of test plate.

\subsection{Repeatability of curvature measurement}

To evaluate the repeatability of the setup, the deviation of the change in curvature was examined under the conditions shown in Table 1 while electrodeposition was not being conducted. The electrolytic cell was filled with nickel sulfamate solution. To eliminate the effect of temperature changes during the measurement, linear components were subtracted. From the relation between the curvature and time shown in Fig. 6, the standard deviation $\Delta \kappa_{S}$ is $0.00026 \mathrm{~m}^{-1}$.

Table 1. Experimental conditions.

\begin{tabular}{cc} 
Solution composition & $\mathrm{Ni}\left(\mathrm{NH}_{2} \mathrm{SO}_{3}\right)_{2} 2.8 \mathrm{~mol} / \mathrm{L}$ \\
Temperature & $23-24{ }^{\circ} \mathrm{C}$ \\
Acquisition time & $0.02 \mathrm{~s}$ \\
Measurement interval & $1 \mathrm{~min}$ \\
Recording time & $3 \mathrm{~h}$ \\
\hline
\end{tabular}

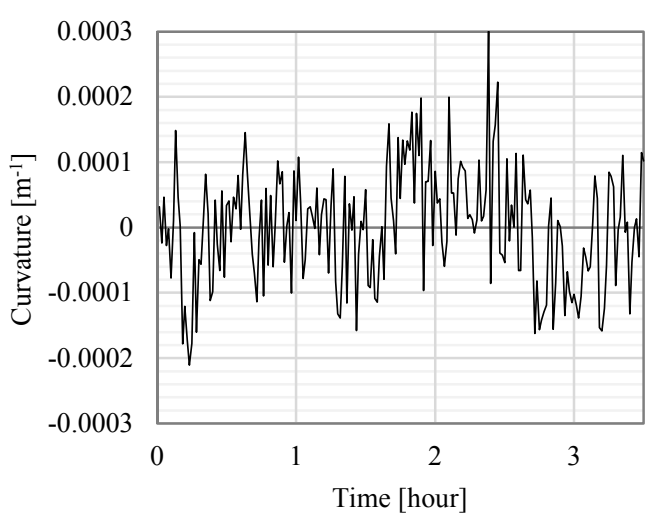

Fig. 6. Relation between curvature of substrate and time without electrodeposition.

\subsection{Accuracy evaluation}

The measurement accuracy was evaluated by comparing the curvatures measured by the proposed setup in concentrated nickel sulfamate solution and a contour-measuring instrument (SURFCOM 200B, Tokyo Seimitsu). The substrate was prepared by conducting electrodeposition under the conditions shown in Table 2. First, to eliminate the effect of aberrations induced in the optical system excluding the bent substrate, the wavefront reflected at a flat substrate placed in the cell was measured as a reference wavefront. Then, the substrate was replaced with the bent substrate. After the wavefront was measured, the reference wavefront was subtracted from the measured wavefront and the curvature of the bent substrate was calculated. Finally, the surface profile of the bent substrate was measured over a length of $60 \mathrm{~mm}$ by the contourmeasuring instrument. The profile of the substrate obtained by the contour-measuring instrument is shown in Fig. 7 (a). The profiles of the substrate 
measured by both methods are shown in Fig. 7 (b), in which $Z_{1}$ and $Z_{2}$ denote the profiles calculated from the curvature obtained by the proposed setup and calculated from a least-squares fitting circle of the profile i $n$ Fig. 7 (a), respectively. The radii of curvature of $Z_{1}$ and $Z_{2}$ were $17.888 \mathrm{~m}$ and $17.525 \mathrm{~m}$, respectively. The difference between these curvatures was $0.0012 \mathrm{~m}^{-1}$. Assuming uniform thickness of the nickel layer and a current efficiency of $100 \%$, the stresses calculated from $Z_{1}$ and $Z_{2}$ were $52 \mathrm{MPa}$ and $53 \mathrm{MPa}$, respectively. The wavefront error is sufficiently small for accuracy of $1 \mathrm{MPa}$ order when the thickness of the electrodeposited layer is over $22 \mu \mathrm{m}$.

Another possible factor that decreases the measurement accuracy is thickness fluctuation. Figs. 8 (a) and (b) show the thickness distributions of the nickel layer obtained along the $x$-axis and $y$-axis, where $y=7.5 \mathrm{~mm}$ and $x=30 \mathrm{~mm}$, respectively. In this method, the stress strongly depends on the cross-sectional profile of the area where the laser beam is reflected. From Fig. 8 (b), the actual cross-sectional area $A_{N O}$ and the actual second moment of the area of the electrodeposited layer $I_{N 0}$ are $89 \%$ of $A_{N}$ and $79 \%$ of $I_{N}$, respectively. Therefore, the actual stress is $60 \mathrm{MPa}$ and the measurement accuracy is approximately $8 \mathrm{MPa}$.

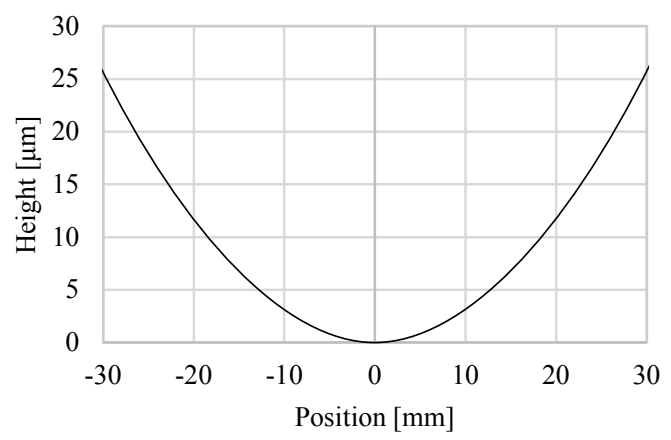

(a) Profile of substrate measured by contour-measuring instrument.

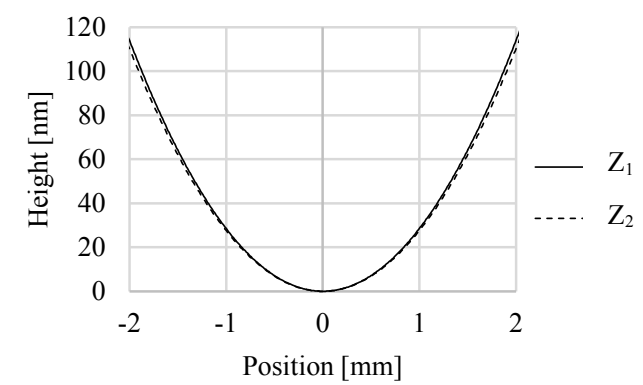

(b) Comparison of curvature.

Fig. 7. Measured profiles of substrate.
Table 2. Experimental conditions.

\begin{tabular}{ll}
\hline Solution composition & $\mathrm{Ni}\left(\mathrm{NH}_{2} \mathrm{SO}_{3}\right)_{2} \quad 2.8 \mathrm{~mol} / \mathrm{L}$ \\
Temperature & $25{ }^{\circ} \mathrm{C}$ \\
Current density & $3.0 \mathrm{~mA} / \mathrm{cm}^{2}$ \\
Electrodeposition time & $5.8 \mathrm{~h}$ \\
Thickness of Ni layer & $22 \mu \mathrm{m}$ \\
\hline
\end{tabular}

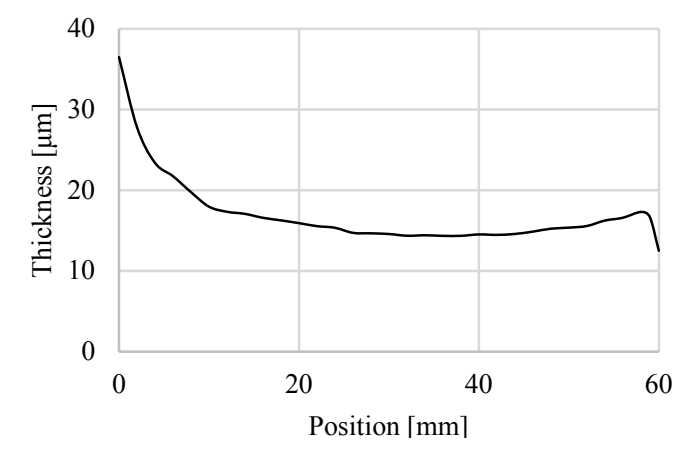

(a) Thickness of Ni layer along $x$-axis.

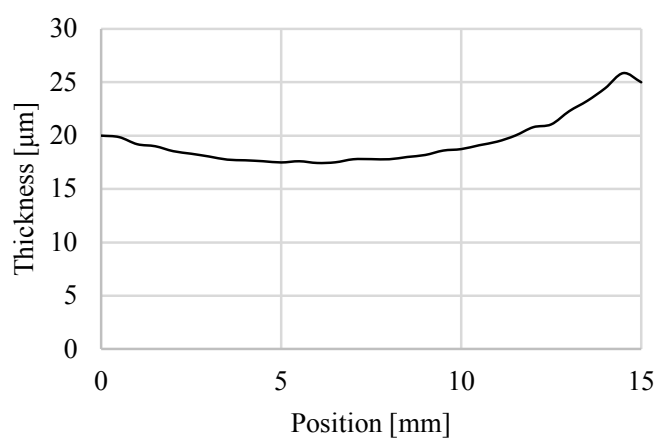

(b) Thickness of Ni layer along $y$-axis.

Fig. 8. Thickness fluctuation of Ni layer.

\section{MEASUREMENT UNDER PRACTICAL CONDITIONS}

During electrodeposition, hydrogen bubbles are also generated on a cathode because the equilibrium potential of nickel deposition in nickel sulfamate solution $\left(\left[\mathrm{Ni}^{2+}\right]=2.8 \mathrm{~mol} / \mathrm{L}, \mathrm{pH} 5.00,25^{\circ} \mathrm{C}\right)$ is close to that of the hydrogen generation reaction. The bubbles adhere to the surface of the electrodeposited nickel layer when the surface tension is high. In the conventional electroforming process with a heated solution $\left(40-60{ }^{\circ} \mathrm{C}\right)$, bubbles are removed by adding a surfactant or agitating the solution. At room temperature, however, it is difficult to dissolve a sufficient amount of surfactant and periodic degassing is effective for removal of the bubbles ${ }^{11)}$. 
The repeatability of the measurement was estimated. Table 3 shows the experimental conditions. The electrolytic cell was connected to a vacuum pump and degassed to $0.02 \mathrm{MPa}$ for $5 \mathrm{~min}$ of every hour. The growth rate of the electrodeposited layer was $2.58 \mu \mathrm{m} / \mathrm{h}$. The measurement was conducted twice using the same solution. The recording interval was $10 \mathrm{~min}$ and a current density of $2.1 \mathrm{~mA} / \mathrm{cm}^{2}$ was applied in both measurements.

The change in the stress during electrodeposition measured in each experiment is shown in Fig. 9, where tensile stress is expressed as positive values. The periodic fluctuations resulted from the deformation of the cell caused by periodic degassing. To eliminate the effect, the relation between the stress obtained when the pressure in the cell returned to atmospheric pressure and the thickness of the deposited layer is shown in Fig. 10. Initially, the stresses in the two measurements are different. One reason for this is that, according to equation (6), the stress is easily affected by the noise in the wavefront when $A_{N}$ is small. In addition, the initial stress is generally induced by the mismatch between the lattices of the electrodeposited metal and the electrode surface. Differences in the vapor deposition conditions or the oxidation of the electrode layer before the experiments are a possible reason for the difference in the initial stresses. From the practical viewpoint, however, the stress in the nickel layer with a thickness of $10 \mu \mathrm{m}$ order is important. The stresses in the measurements were $32 \mathrm{MPa}$ and $33 \mathrm{MPa}$ when the thicknesses were $19.7 \mu \mathrm{m}$ and $18.8 \mu \mathrm{m}$, respectively. The repeatability of the stress measurement after the thickness reached $15 \mu \mathrm{m}$ was $1 \mathrm{MPa}$. Using the apparatus, a measurement with a current density of $0.7 \mathrm{~mA} / \mathrm{cm}^{2}$ was also conducted. As shown in Fig. 11, compressive stress was observed. The high-frequency change observed in the range of $20-25 \mu \mathrm{m}$ is noise caused by hydrogen bubbles adhered to the glass substrate.

It is considered that the measurement apparatus will be useful under practical conditions for room-temperature electroforming.

Table 3. Experimental conditions.

\begin{tabular}{ll}
\hline Solution composition & $\mathrm{Ni}\left(\mathrm{NH}_{2} \mathrm{SO}_{3}\right)_{2} 2.8 \mathrm{~mol} / \mathrm{L}$ \\
Temperature & $25{ }^{\circ} \mathrm{C}$ \\
Current density & $2.1 \mathrm{~mA} / \mathrm{cm}^{2}$ \\
Electrodeposition time & $7.3 \mathrm{~h}, 7.8 \mathrm{~h}$ \\
Thickness of Ni layer & $19 \mu \mathrm{m}, 20 \mu \mathrm{m}$ \\
\hline
\end{tabular}

\section{CONCLUSION}

In this paper, the performance of a stress measurement method for nickel electroforming using an SHS was examined. An accuracy of approximately $8 \mathrm{MPa}$ and a repeatability of $1 \mathrm{MPa}$ under practical conditions for electroforming were confirmed when the thickness fluctuation was considered.

It is possible to improve the accuracy to $1 \mathrm{MPa}$ or der by calibrating $A_{N}$ with information on the thickness fluctuation of the nickel layer. Further improvement in the measurement performance can also be expected by constructing a more rigid cell and stabilizing the measurement environment.

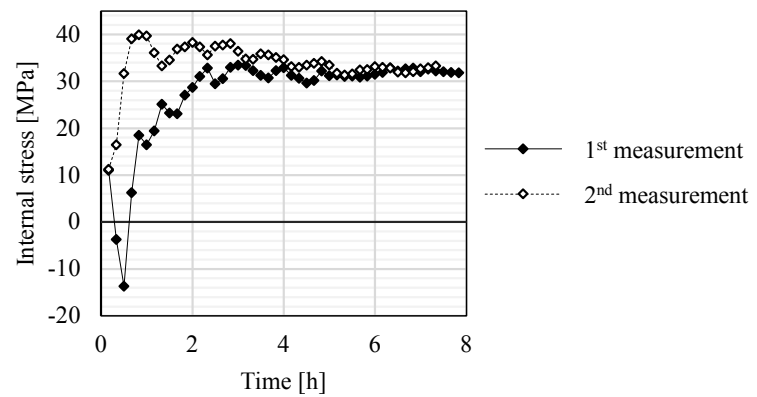

Fig. 9. Change in stress during electrodeposition with current density of $2.1 \mathrm{~mA} / \mathrm{cm}^{2}$.

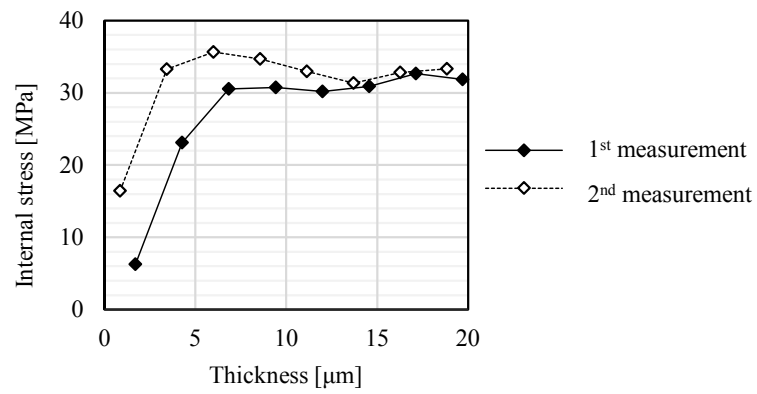

Fig. 10. Relation between internal stress and thickness during electrodeposition with current density of $2.1 \mathrm{~mA} / \mathrm{cm}^{2}$.

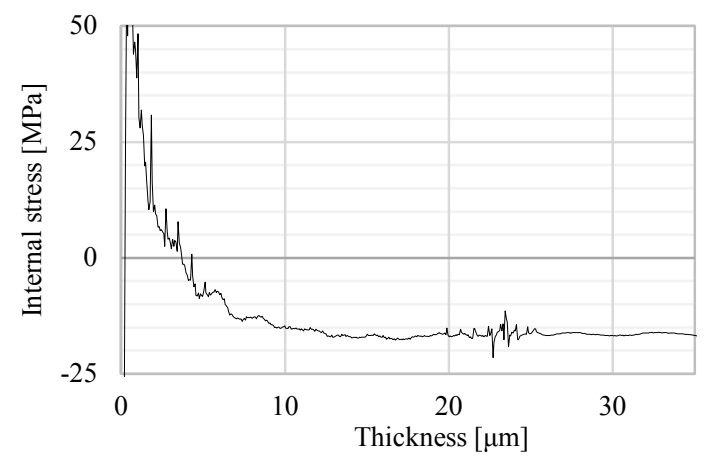

Fig. 11. Change in stress during electrodeposition with current density of 0.7 $\mathrm{mA} / \mathrm{cm}^{2}$ 


\section{REFERENCES}

1) McGeough JA. et al., 2001. Electroforming Process and Application to Micro/Macro Manufacturing, CIRP Annals-Manufacturing Technology 50(2), pp. 499-514.

2) Ramsey BD. et al., 2002. First Images from HERO, a Hard X-ray Focusing Telescope, The Astrophysical Journal 568(1), pp. 432-465.

3) Takei Y. et al., 2013. Development of a Numerically Controlled Elastic Emission Machining System for Fabricating Mandrels of Ellipsoidal Focusing Mirrors Used in Soft X-ray Microscopy, Procedings of SPIE 8848, $88480 \mathrm{C}$

4) Stoney GG., 1909. The Tension of Metallic Films Deposited by Electrolysis, Proceedings of the Royal Society of London A82 (553), pp. 172-175.

5) Bush GW. and Read HJ., 1964. X-Ray Determination of Residual Stresses in Electrodeposited Coatings, Journal of the Electrochemical Society 111(3), pp. 289-296.

6) Brenner A. and Seymour S., 1949. A Spiral Contractometer for Measuring Stress in Electrodeposits, Journal of Research of the National Bureau of Standards 42, pp. 89-104.
7) Floro JA. and Chason E., 1996. Measuring Ge Segregation by Real-time Stress Monitoring during $\mathrm{Si}_{1-\mathrm{x}} \mathrm{Ge}_{\mathrm{x}}$ Molecular Beam Epitaxy, Applied Physics Letters 69(25), pp. 3830-3832.

8) Proost J. and Spaepen F., 2002. Evolution of the Growth Stress, Stiffness, and Microstructure of Alumina Thin Films during Vapor Deposition, Journal of Applied Physics 91(1), pp. 204-216.

9) Overmeere QV, Vanhumbeeck JF and Proost J., 2010. On the Use of a Multiple Beam Optical Sensor for In Situ Curvature Monitoring in Liquids, Review of Scientific Instruments 81(4), 045106.

10) Egawa S., Kume T. and Mimura H., 2014. Improvement of Electroforming by Deflection Measurement with a Shack-Hartmann Sensor, Proceedings of 2014 JSPE Spring Meeting, pp.1273-1274.

11) Kume T., Egawa S. and Mimura H., 2014. Development of Nanometer-level Accurate Replication Process Using Electroforming -Optimization of Room Temperature Nickel Electrodeposition Conditions and Precise Figure Replication of Mandrel-, Journal of the Japan Society for Precision Engineering 80(6), pp.582-586. 